\title{
Unraveling the Molecular Basis for Successful Thyroid Hormone Replacement Therapy: The Need for New Thyroid Tissue- and Pathway-Specific Biomarkers
}

\author{
Authors \\ Sebastian Nock ${ }^{1}$, Carolin Höfig ${ }^{2}$, Lisbeth Harder ${ }^{1}$, Lutz Schomburg ${ }^{2}$, Georg Brabant ${ }^{1}$, Jens Mittag1
}

\section{Affiliations}

1 Medizinische Klinik I, Universitätsklinikum SchleswigHolstein, Lübeck, Germany

2 Institute for Experimental Endocrinology, Charité -Universitaetsmedizin Berlin, Berlin, Germany

Keywords

proteome, metabolome, transcriptome, hypothyroidism, hyperthyroidism, selenium, copper

$\begin{array}{ll}\text { received } & 08.08 .2019 \\ \text { revised } & 09.09 .2019 \\ \text { accepted } & 12.09 .2019\end{array}$

\section{Bibliography}

DOI https://doi.org/10.1055/a-1012-8484

Published online: 7.10.2019

Exp Clin Endocrinol Diabetes 2020; 128: 473-478

(C) J. A. Barth Verlag in Georg Thieme Verlag KG Stuttgart . New York

ISSN 0947-7349

Correspondance

Prof. Dr. Jens Mittag

Medizinische Klinik I

Universitätsklinikum Schleswig-Holstein

Ratzeburger Allee 160

23562 Lübeck

Germany

Tel.: + 49/451/31017 826,

jens.mittag@uni-luebeck.de

\begin{abstract}
Thyroid function is conventionally assessed by measurement of thyroid-stimulating hormone (TSH) and free circulating thyroid hormones, which is in most cases sufficient for correct diagnosis and monitoring of treatment efficiency. However, several conditions exist, in which these parameters may be insufficient or even misleading. For instance, both, a TSH-secreting pituitary adenoma and a mutation of thyroid hormone receptor $\beta$ present with high levels of TSH and circulating hormones, but the optimal treatment is substantially different. Likewise, changes in thyroid hormone receptor $\alpha$ signaling are not captured by routine assessment of thyroid status, as serum parameters are usually inconspicuous. Therefore, new biomarkers are urgently needed to improve the diagnostic management and monitor treatment efficiency for e. g., replacement therapy in hypothyroidism or thyroid hormone resistance. By comparing animal models to human data, the present minireview summarizes the status of this search for new tissue- and pathway-specific biomarkers of thyroid hormone action.
\end{abstract}

\section{Introduction}

Hypothyroidism is a frequent condition with an estimated prevalence of $1.5 \%$ in the northern and $4.1 \%$ in the southern parts of Germany [1]. It is conventionally treated by oral monotherapy with levothyroxine (T4) as supported by all guidelines on hypothyroidism [2-4]. Monotherapy with T4 as a prohormone is both possible and safe, because it is converted intracellularly in target tissues to the biologically active 3,3',5-triiodothyronine (T3). T3 binds with high affinity to the nuclear thyroid hormone $(\mathrm{TH})$ receptors TR $\alpha$ and $T R \beta$, which are encoded by the two separate genes THRA and
THRB. TRs positively or negatively regulate TH dependent gene expression in almost all tissues, providing the molecular explanation for the plethora of symptoms observed in thyroid disorders [5]. Tissues and cells can however to a large extent regulate their TH action independently of the serum concentrations, as in addition to TRs other control layers exist. The first prerequisite for TH action is the cell- and hormone-specific uptake of T3 and/or T4 by transmembrane transporters. Secondly, subsequent intracellular conversion of TH is necessary, facilitated by a family of activating enzymes, the selenium (Se) dependent deiodinases (DIOs). Type 1 
(DIO1) and type 2 (DIO2) deiodinases are capable of generating bioactive $\mathrm{T} 3$ from T4, whereas type 3 (DIO3) acts as an inactivating enzyme [6]. Therefore, time- and tissue-specific alterations in the expression of any member of this regulatory network contribute to explain why local TH action can differ between target organs despite comparable and relatively constant circulating TH levels and why there are distinct differences between species despite a high evolutionary conservation of $\mathrm{TH}$ activation and $\mathrm{TH}$ receptors [5]. Consequently, it is possible that tissues display features of hypo- or hyperthyroidism, although the serum profile of $\mathrm{TH}$ is in the euthyroid range. This variability of local TH action can occur physiologically, e.g., by an upregulation of DIO activity in certain periods of development [7], as part of a pathological condition, for instance a downregulation of THRB expression in human non-alcoholic steatohepatitis [8] or during non-thyroidal illness syndrome [9], or as a consequence of inactivating mutations in TH transporters or receptors $[10,11]$. As these conditions cannot be assessed by serum $\mathrm{TH}$ concentrations, additional circulating biomarkers are urgently required that provide information about the local TH action in a specific tissue, e. g., the liver.

\section{Diagnostic Approach and Current Limitations}

Currently, the accepted gold standard to diagnose primary hypothyroidism and to monitor thyroid status under treatment is to measure serum thyrotropin (TSH), a hormone produced in a $\mathrm{TH}$ and TR $\beta$-dependent manner in the anterior pituitary [6]. This parameter is often superior to the measurement of serum free or total $\mathrm{TH}$, which may be subject to a number of interfering factors such as the duration and severity of the underlying thyroid disease $[12,13]$. In addition, measurement of $\mathrm{TH}$, particularly of free serum T3 or T4 levels, is methodologically demanding and vulnerable to various interferences e. g., by drugs or structurally similar endocrine disrupting compounds due to the very low serum concentrations of T3 [14]. Guidelines therefore advice against drawing firm conclusions from minor alterations in circulating TH levels, but accept TSH as the best single, robust, sensitive, and reproducible test for determining thyroid function [15]. Yet, several conditions are known, where relying on TSH serum concentrations are misleading. For instance, in central hypothyroidism the normal glycosylation of TSH is reduced leading to a partial or complete loss of bioactivity, while clinical assays measuring TSH would not detect any differences due to a preserved immunoreactivity of the less glycosylated protein [16]. TSH may thus still be inadequately maintained within the normal range despite low circulating $\mathrm{TH}$. As another example, a TSH-secreting pituitary tumor (TSHoma) presents with high levels of circulating TSH and consequently highly elevated circulating TH levels, which fail to suppress TSH expression. Using solely the serum profile, this condition is impossible to distinguish from inactivating mutations in TR $\beta$ (resistance to thyroid hormone $\beta, \operatorname{RTH} \beta,[17])$, which impair the TR $\beta$-controlled suppression of pituitary TSH expression by TH and also presents with inappropriately high TSH serum concentrations for the corresponding TH levels [18]. Consequently, the current standard of TSH measurement is clearly insufficient under certain pathological conditions to faciliate a correct diagnosis.
Likewise, even in more common conditions such as substitution with T4 in hypothyroidism, monitoring of thyroid function with TSH and TH concentrations fails, because serum total or free T3 and T4 will vary with the timing and dose of T4 replacement [4]. In addition, serum TSH may take several weeks to normalize in severe hypo- or hyperthyroidism. Furthermore, in a cohort of almost 2000 athyroid patients studied under $\mathrm{T} 4$ replacement therapy more than $20 \%$ did not maintain their free T4 and T3 levels within the reference range and almost $30 \%$ showed an abnormally low fT3/fT4 ratio. Interestingly, the gradient between TSH and fT3 increased with the dose of L-T4, indicating that TSH may not be sufficient for assessing euthyroidism $[19,20]$. Moreover, it is still controversially discussed whether it is required to titrate TSH back to the optimal range, as TSH might also be involved in the regulation of extrathyroidal tissues expressing the TSH receptor, e. g., kidney or bone [21]. The unreliability of TSH as treatment progression marker is unfortunate, as impaired psychological well-being, depression or anxiety can still be present in $5-10 \%$ of patients despite normal TSH levels under standard T4 substitution [22], and the resolution of hypothyroid symptoms is one of the most important targets of TH replacement therapy in hypothyroidism [4]. Likewise, biological markers indicative of hypothyroidism such as heart rate, pulse wave arrival time, echocardiographic parameters of left ventricular function, Achilles reflex time, voice fundamental frequency, or basal metabolic rate are not sensitive enough to be used in the diagnosis or follow-up of therapy in hypothyroidism due to their high variability and their lack of sensitivity and/or specificity [4].

\section{Alternative Biomarkers}

To overcome these problems with the key diagnostic markers to assess thyroid status, other biochemical markers have been tested. The liver-derived sex hormone-binding globulin (SHBG), another TR $\beta$-dependent protein, is of some clinical importance under certain conditions, but its specificity is reduced due to the responsiveness to other non-thyroidal factors such as sex hormones [23]. A similar modulation by non-thyroidal regulators has also been described for other potential biochemical markers of TH status such as lipid parameters (total cholesterol, LDL cholesterol, lipoprotein(a)), bone markers (osteocalcin, urinary n-telopeptides (NTX)) or creatine kinase, ferritin, myoglobin, tissue plasminogen activator, ACE, and glucose 6-phosphate dehydrogenase [24]. These limitations resulted in guideline recommendations that "tissue biomarkers of thyroid hormone action are not recommended for routine clinical use, outside of the research setting, since these parameters are not sensitive, specific, readily available, or standardized" or "would require invasive procedures such as tissue biopsy" [4]. Therefore, they are not part of the routine work-up of patients, although SHBG is occasionally used clinically as an additional biomarker, for instance in the aforementioned differential diagnosis of an inappropriately elevated TSH. Here, elevated SHBG levels indicate liver thyrotoxicosis and point to TSHoma, whereas in RTH $\beta$ the liver is partially protected from the effects of the high circulating TH due to the impaired TR $\beta$ signaling, and SHBG levels would not be increased. 

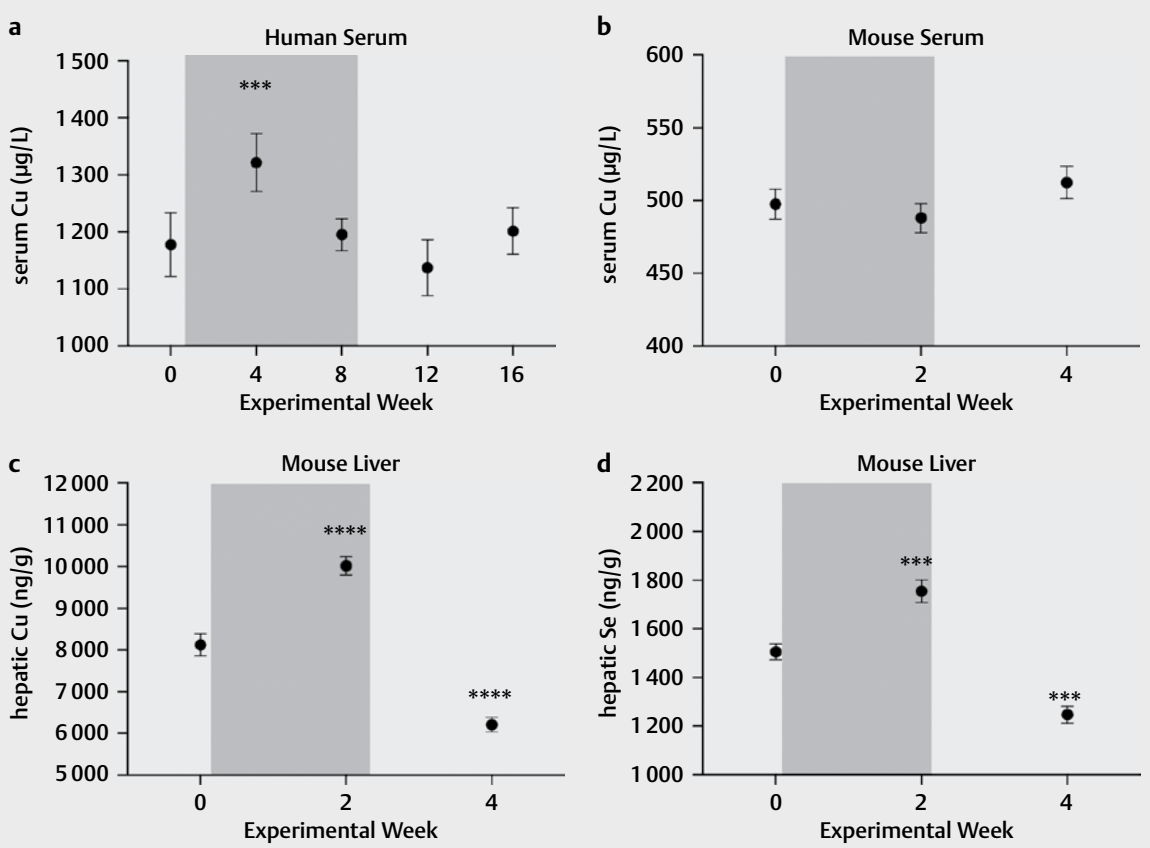

- Fig. 1 a Serum Cu concentrations in human volunteers exposed for 8 weeks to a mild T4-induced thyrotoxicosis (grey box) and subsequent recovery (31). b Serum Cu concentrations in mice treated with a comparable dose of T4. $\mathbf{c}+\mathbf{d}$ Hepatic $\mathrm{Cu}$ and Se content in the animals from $\mathbf{b}$. Values depict mean \pm SEM, $p<0.001:{ }^{* *} ; \mathrm{p}<0.0001:{ }^{* * *}$ (repeated measure ANOVA with paired a or non-paired samples $\mathbf{b}$-d), corrected for multiple comparison with Holm-Sidak test). Se and Cu were measured using a picofox instrument as published previously [25, 26].

\section{Selenium and Copper as Novel Biomarkers}

More recent studies in humans and animal models with RTH $\beta$ suggested that the trace elements Se and copper (Cu) could be helpful in the differential diagnosis between TSHoma and RTH $3[25,26]$, as the T3-induced increase in serum Cu depends on hepatic TR $\beta$ action. Consequently, in mice lacking TR $\beta$ and individuals with RTH $\beta$, the serum Cu levels were reduced, as despite the elevated serum TH, the liver is in a hypothyroid state due to the lack of TR $\beta$ action. In contrast, serum Cu was similarly elevated by $T 3$ treatment in control mice as well as animals expressing a mutant TR $\alpha 1$, demonstrating the TR $\beta$ - but not TR $\alpha 1$-dependency of this regulation [26]. Most interestingly, serum Se was elevated in RTH $\beta$ and T3-treated animals, but lower in mice expressing a mutant TR $\alpha 1$, suggesting a TR $\alpha 1$-dependent regulation of this trace element $[25,26]$. Since data from hyperthyroid humans have not been available, we analyzed the serum Se and Cu levels now in our experimental thyrotoxicosis model in humans [27]. The results showed an induction of serum Cu after 4 but not 8 weeks of T4 treatment ( $\vee$ Fig 1a), suggesting a quick response to TH but compensation after a longer period of hyperthyroidism. Serum Se levels did unexpectedly not change significantly (not shown). Interestingly, the induction of Cu was not observed after 2 weeks treatment with a comparable dose of T4 in mice ( $\vee$ Fig $\mathbf{1 b}$ ); however, levels of Se and $\mathrm{Cu}$ in the livers of these animals increased markedly $(\triangleright \mathbf{F i g} \mathbf{1} \mathbf{c}+\mathbf{d})$. This contrasts with previous studies showing elevated serum $\mathrm{Cu}$ concentrations, but reduced hepatic Cu content after 2 weeks treatment with a high dose of T3 [26], which could indicate that TH initially and at lower doses elevates hepatic Cu storage, while high- er doses also trigger the release from the liver e. g., as ceruloplasmin as demonstrated previously [26]. Together with recent studies showing a strong correlation between serum $\mathrm{TH}$ and $\mathrm{Cu}$ concentrations in children with congenital hypothyroidism [28] or even in larger epidemiological studies in the United States [29], these observations support a positive regulation of serum $\mathrm{Cu}$ by $\mathrm{TH}$. However, before these findings can be translated to clinical use to assess hepatic (TR $\beta$ dependent) TH action in humans, additional clinical studies are required to substantiate these findings, their possible responsiveness to non-thyroidal factors, their time- and dose dependency as well as the species-specific differences.

\section{Perspectives for New Biomarkers With Better Sensitivity to Monitor Local TH Action}

Given that measurement of TSH serum levels fail in a number of important pathophysiological conditions to adequately represent thyroid function and the direct measurement of TH and/or of the available tissue biomarkers is associated with other major limitations in routine use [15], additional sensitive means to diagnostically capture the local action of $\mathrm{TH}$ in selected tissues are urgently needed to improve the assessment of euthyroidism and guide pharmacological treatment in common ( $\mathrm{T} 4$ replacement therapy in hypothyroidism) as well as rare (inactivating mutations in TR $\alpha, T R \beta$, TH transporters) conditions. Given the sensitivity of several biomarkers to non-thyroidal regulators, it is unlikely that a single biomarker will be sufficient for routine clinical use. Therefore, recent 
strategies employing an unbiased multifactorial approach, summarized as "OMICs" techniques for the common ending in targets like genomics, proteomics and metabolomics, may be suited to capture the pleiotropic effects of $\mathrm{TH}$. The development of high throughput analytical platforms with better sensitivity and precision increasingly allows applying these techniques in many diagnostic fields, and software packages may be able to extract a $\mathrm{TH}$ dependent fingerprint of tissue-selective biomarkers. To date, only few studies on OMIC analyses in blood in relation to thyroid function have been performed, and within-subject studies before and following T4 withdrawal revealed almost 500 differentially regulated targets [30]. Another study investigated patients with overt hypothyroidism at the time of diagnosis and after serum TSH levels had been normalized for at least 6 weeks under T4 substitution therapy and identified 20 differentially regulated protein spots [31]. Unfortunately, the heterogeneity of the patient group is a major factor interfering with the reliability of the results, as especially gender strongly modulates the effects of $\mathrm{TH}$ treatment. In a within-subject study we aimed to avoid these problems by inducing hyperthyroidism experimentally in male volunteers only [27]. Interestingly, despite clear biochemical changes fitting to thyrotoxicosis 4 and 8 weeks under T4 treatment, none of the subjects developed any clinical sign or any significant change in thyroid specific symptoms (with the exception of a nightly increase in heart rate by an average of $6 \mathrm{bpm}$ ), neither as compared to baseline before treatment nor as compared to the final state following 8 weeks after the treatment. These results support the previously described dissociation between biochemical tests for thyroid function and symptoms as seen in cross-sectional studies [27, 32]. Subsequent proteome analysis, conducted in collaboration with our partners in Greifswald, revealed a significant $\mathrm{TH}$ status associated change in several protein signatures, namely in the abundance of apolipoproteins (decreased APOD, APOB and APOC-III levels in the thyrotoxic state) and of proteins related to the coagulation cascade and the complement system (increased levels). Especially several coagulation factors, namely II, V, XI and XIII were increased shortly after the induction of thyrotoxicosis [33], likely reflecting a TR $\beta$-dependent regulation as direct testing in RTH $\beta$ patients confirmed an unchanged balance in the coagulation factors [34]. However, another study using a 2 week experimental treatment period with T4 in healthy subjects did not observe alterations in the complement system or blood clotting [35], suggesting that the timing of thyroid dysfunction appears to be important and the response curve is most likely $U$-shaped, as the proteome indicated an increased activity of these factors in hypothyroidism as well [31].

Consequently, to unravel the underlying molecular mechanisms additional studies will be needed, including animal models with impaired local TH action, i. e. animal models for RTH $\alpha$ or RTH $\beta$ $[25,26]$ as well as pharmacological studies [36]. The majority of existing data from animal models are mainly derived from the comparison of hypothyroidism to the euthyroid state in rodents $[37,38]$, which may not be of biological relevance for other conditions. Moreover, there are some discrepancies to the human situation especially with regard to the size of biological effects such as heart rate or body temperature $[27,36]$. Furthermore, the direct comparison of OMICs based biomarkers between mice and man is hampered by technical problems, as e. g., many of the most abun- dant proteins are different, thus impacting on the rate of recovery of other rare peptides and proteins. Despite these possible problems, parallel and discrepant regulation can be distinguished when comparing the two species. For the coagulation system, hyperthyroidism in humans is associated with an increased clot formation and a higher risk of venous thromboembolism whereas coagulability in mice appears to be reduced [39]. Other systems like the apolipoproteins were reduced in parallel between mice and humans. In our interspecies comparison study between human and mice $[27,36]$, we have already identified 16 serum proteins that are concordantly altered by $\mathrm{T} 4$ in both species, including a macrophagederived protein [40]. This demonstrates that such an approach has great potential to not only reveal a robust set of TH dependent biomarkers, but also to understand the underlying regulatory mechanisms and identify the involved components of the TH regulatory network or non-thyroidal regulators.

Despite of discrepancies in proteome analysis, which may largely depend on the methodological uncertainties, most of the pleotropic effects of $\mathrm{TH}$ would be expected in the regulation of endogenous metabolic pathways. Therefore, techniques capturing the small molecules comprising the metabolome have been used to identify changes in more abundant metabolites such as carbohydrates, lipids, amino acids and alterations in some low concentration molecules like organic acids [24], showing a different signature in association to serum TSH or T4 [41], which was partially confirmed in an independent study [42]. Most recently, a study in 4 different animal models of RTH $\alpha$ demonstrated that using NMR, a clear metabolic fingerprint of the disease in biofluids could be identified, which was stable over time and dinstinctly different from hypothyroidism [43]. These initial findings in humans and rodents suggests that despite the fact that many metabolites are variably regulated by several different conditions, TH may still leave a detectable shift in the overal metabolomic pattern, which could be used as a putative diagnostic tool. However, as almost all of these metabolites are highly variable and by nature even more affected by non-thyroidal regulators or external factors such as food, circadian rhythms or drugs than protein biomarkers, it remains to be seen whether a metabolomic fingerprint can be clinically useful. Moreover, the subtle differences in the induction of hyper- or hypothryoidism in rodent studies regarding route of administration and dose also need to be considered as variable, although recently defined guidelines for these experimental protocols have been suggested [44].

\section{Perspectives}

The results of our experimental studies in mice and humans provided novel molecular insight, which due to the tightly controlled conditions, are likely to represent pure T4-dependent effects. This combined approach in animal models and humans also allowed assessing organ- and TR-specific TH effects, for instance the TR $\beta$ mediated regulation of hepatic Cu metabolism. If these initial findings can be validated successfully, direct, specific and highly sensitive assays with high discriminatory power may be developed in the future to establish new and more targeted biomarkers providing valuable clinical information about the local TH action. 


\section{Quick Summary}

This minireview summarizes the status of the search for new tissue- and pathway-specific biomarkers reflecting local thyroid hormone action.

\section{Acknowlegdements}

The authors are grateful for technical support from Vartitér Seher and Sandra Hybsier, and acknowledge funding from the German Research Council DFG in the framework program SPP1629 "Thyroid TransAct” (MI1242/5-1, BR915/12-1, HO5096/2-1), the DFG Reseach Unit 2558 TraceAge (SCHO849/6-1), and the Heisenberg Program (MI1242/2-1 and 2/2). We thank our colleagues in Greifswald for the collaboration on the Omics data and refer to their respective review in this special issue for further details regarding circulating biomarkers in humans.

\section{Conflict of Interest}

The authors declare that they have no conflict of interest.

\section{References}

[1] Meisinger C, Ittermann T, Wallaschofski H et al. Geographic variations in the frequency of thyroid disorders and thyroid peroxidase antibodies in persons without former thyroid disease within Germany. Eur J Endocrinol. / European Federation of Endocrine Societies 2012; 167: 363-371. doi:10.1530/EJE-12-0111

[2] Jonklaas ], Bianco AC, Bauer A] et al. Guidelines for the treatment of hypothyroidism: Prepared by the american thyroid association task force on thyroid hormone replacement. Thyroid 2014; 24: 1670-1751. doi:10.1089/thy.2014.0028

[3] Pearce SH, Brabant G, Duntas LH et al. 2013 ETA Guideline: Management of Subclinical Hypothyroidism. Eur Thyroid J 2013; 2: 215-228. doi:10.1159/000356507

[4] Wiersinga WM, Duntas L, Fadeyev V et al. 2012 ETA Guidelines: The use of L-T4 + L-T3 in the treatment of hypothyroidism. Eur Thyroid J 2012; 1: 55-71. doi:10.1159/000339444

[5] Mendoza A, Hollenberg AN. New insights into thyroid hormone action. Pharmacol Therapeut 2017; 173: 135-145. doi:10.1016/j. pharmthera.2017.02.012

[6] Yen PM. Physiological and molecular basis of thyroid hormone action. Physiol Rev 2001; 81: 1097-1142

[7] Fonseca TL, Fernandes GW, McAninch EA et al. Perinatal deiodinase 2 expression in hepatocytes defines epigenetic susceptibility to liver steatosis and obesity. Proceedings of the National Academy of Sciences of the United States of America 2015; 112: 14018-14023. doi:10.1073/pnas.1508943112

[8] Krause C, Grohs M, El Gammal A et al. Reduced expression of thyroid hormone receptor beta in human nonalcoholic steatohepatitis. Endocrine Connections 2018, doi:10.1530/EC-18-0499

[9] de Vries EM, Fliers E, Boelen A. The molecular basis of the non-thyroidal illness syndrome. J Endocrinol 2015; 225: R67-R81

[10] Bochukova E, Schoenmakers N, Agostini M et al. A mutation in the thyroid hormone receptor alpha gene. N Engl J Med 2012; 366: 243-249
[11] Friesema EC, Grueters A, Biebermann $\mathrm{H}$ et al. Association between mutations in a thyroid hormone transporter and severe X-linked psychomotor retardation. Lancet 2004; 364: 1435-1437. doi:10.1016/ S0140-6736(04)17226-7

[12] Biondi B, Cooper DS. Subclinical Hyperthyroidism. N Engl J Med 2018; 378: 2411-2419. doi:10.1056/NEJMcp 1709318

[13] Peeters RP. Subclinical Hypothyroidism. N Engl J Med 2017; 377: 1404 doi:10.1056/NEJMc1709853

[14] Koulouri O, Moran C, Halsall D et al. Pitfalls in the measurement and interpretation of thyroid function tests. Best Pract Res Clin Endocrinol Metab 2013; 27: 745-762

[15] Baloch Z, Carayon P, Conte-Devolx B et al. Laboratory medicine practice guidelines. Laboratory support for the diagnosis and monitoring of thyroid disease. Thyroid 2003; 13: 3-126. doi:10.1089/105072503321086962

[16] Persani L, Brabant G, Dattani M et al. 2018 European Thyroid Association (ETA) Guidelines on the diagnosis and management of central hypothyroidism Eur. Thyroid J 2018; 7: 225-237. doi:10.1159/000491388

[17] Refetoff S, DeWind LT, DeGroot LJ. Familial syndrome combining deaf-mutism, stuppled epiphyses, goiter and abnormally high PBI: possible target organ refractoriness to thyroid hormone. J Clin Endocrinol Metab 1967; 27: 279-294

[18] Pappa T, Refetoff S. Human Genetics of Thyroid Hormone Receptor Beta: Resistance to Thyroid Hormone Beta (RTHbeta). Methods in Molecular Biology 2018; 1801: 225-240. doi:10.1007/978-1-49397902-8_18

[19] Gullo D, Latina A, Frasca F et al. Levothyroxine monotherapy cannot guarantee euthyroidism in all athyreotic patients. PloS One 2011; 6: e22552. doi:10.1371/journal.pone.0022552

[20] Hoermann R, Midgley JE, Larisch R et al. Is pituitary TSH an adequate measure of thyroid hormone-controlled homoeostasis during thyroxine treatment? Eur J Endocrinol / European Federation of Endocrine Societies 2013; 168: 271-280. doi:10.1530/EJE-12-0819

[21] Williams G. Extrathyroidal expression of TSH receptor. Ann Endocrinol (Paris) 2011; 72: 68-73

[22] Wiersinga WM. Paradigm shifts in thyroid hormone replacement therapies for hypothyroidism. Nature reviews Endocrinology 2014; 10 : 164-174. doi:10.1038/nrendo.2013.258

[23] Thaler MA, Seifert-Klauss V, Luppa PB. The biomarker sex hormonebinding globulin - from established applications to emerging trends in clinical medicine. Best Pract Res Clin Endocrinol Metab 2015; 29 : 749-760. doi:10.1016/j.beem.2015.06.005

[24] Pietzner M, Kacprowski T, Friedrich N. Empowering thyroid hormone research in human subjects using OMICs technologies. J Endocrinol 2018; 238: R13-R29. doi:10.1530/JOE-18-0117

[25] Mittag J, Behrends T, Hoefig CS et al. Thyroid hormones regulate selenoprotein expression and selenium status in mice. PloS One 2010; 5: e12931

[26] Mittag J, Behrends T, Nordstrom $\mathrm{K}$ et al. Serum copper as a novel biomarker for resistance to thyroid hormone. Biochem J 2012; 443: 103-109

[27] Pietzner M, Engelmann B, Kacprowski T et al. Plasma proteome and metabolome characterization of an experimental human thyrotoxicosis model. BMC Medicine 2017; 15: 6. doi:10.1186/s12916-016-07708

[28] Blasig S, Kuhnen P, Schuette A et al. Positive correlation of thyroid hormones and serum copper in children with congenital hypothyroidism. J Trace Elem Med Biol: Organ of the Society for Minerals and Trace Elements 2016; 37: 90-95. doi:10.1016/j.jtemb.2016.05.007

[29] Jain RB. Thyroid function and serum copper, selenium, and zinc in general U.S. population. Biological Trace Element Research 2014; 159 : 87-98. doi:10.1007/s12011-014-9992-9 
[30] Massolt ET, Meima ME, Swagemakers SMA et al. Thyroid state regulates gene expression in human whole blood. J Clin Endocrinol Metab 2018; 103: 169-178. doi:10.1210/jc.2017-01144

[31] Alfadda AA, Benabdelkamel $\mathrm{H}$, Masood A et al. Differences in the plasma proteome of patients with hypothyroidism before and after thyroid hormone replacement: A proteomic analysis. Int J Mol Sci 2018; 19. doi:10.3390/ijms19010088

[32] Feller M, Snel M, Moutzouri E et al. Association of Thyroid Hormone Therapy With Quality of Life and Thyroid-Related Symptoms in Patients with subclinical hypothyroidism: A systematic review and meta-analysis. Jama 2018; 320: 1349-1359. doi:10.1001/ jama.2018.13770

[33] Engelmann B, Bischof J, Dirk AL et al. Effect of experimental thyrotoxicosis onto blood coagulation: A proteomics study Eur Thyroid J 2015; 4: 119-124. doi:10.1159/000381769

[34] Elbers LP, Moran C, Gerdes VE et al. The hypercoagulable state in hyperthyroidism is mediated via the thyroid hormone beta receptor pathway. Eur J Endocrinol / European Federation of Endocrine Societies 2016, doi:10.1530/EJE-15-1249

[35] Hooper JM, Stuijver DJ, Orme SM et al. Thyroid dysfunction and fibrin network structure: A mechanism for increased thrombotic risk in hyperthyroid individuals. J Clin Endocrinol Metab 2012; 97: 1463 1473. doi:10.1210/jc.2011-2894

[36] Hoefig CS, Harder L, Oelkrug R et al. Thermoregulatory and cardiovascular consequences of a transient thyrotoxicosis and recovery in male mice. Endocrinology 2016; 157: 2957-2967. doi:10.1210/en.20161095

[37] Montoya GA, Strauss V, Fabian E et al. Mechanistic analysis of metabolomics patterns in rat plasma during administration of direct thyroid hormone synthesis inhibitors or compounds increasing thyroid hormone clearance. Toxicology Letters 2014; 225: 240-251. doi:10.1016/j.toxlet.2013.12.010
[38] Wu S, Gao Y, Dong X et al. Serum metabonomics coupled with Ingenuity Pathway Analysis characterizes metabolic perturbations in response to hypothyroidism induced by propylthiouracil in rats. J Pharmaceut Biomed 2013; 72: 109-114. doi:10.1016/j. jpba.2012.09.030

[39] Srisawat S, Sitasuwan T, Ungprasert P. Increased risk of venous thromboembolism among patients with hyperthyroidism: A systematic review and meta-analysis of cohort studies. Eur J Intern Med 2019, doi:10.1016/j.ejim.2019.06.012

[40] Nock S, Johann K, Harder L et al. Identification of new thyroid hormone dependent biomarkers for a successful replacement therapy. Endocrine Abstracts 2018; 56: GP253. doi:10.1530/endoabs.56.GP253

[41] Pietzner M, Budde K, Homuth G et al. Distinct urinary metabolic profiles associated with serum TSH and FT4 concentrations. Metabolomics 2015; 11: 1316-1326. doi:https://doi.org/10.1007/ s11306-015-0788-5

[42] Friedrich N, Pietzner M, Cannet C et al. Urinary metabolomics reveals glycemic and coffee associated signatures of thyroid function in two population-based cohorts. PloS One 2017; 12: e0173078. doi:10.1371/journal.pone. 0173078

[43] Boumaza H, Markossian S, Busi B et al. Metabolomic profiling of body fluids in mouse models demonstrates that NMR is a Putative Diagnostic Tool For The Presence Of Thyroid Hormone Receptor alpha1 mutations. Thyroid 2019, doi:10.1089/thy.2018.0730

[44] Bianco AC, Anderson G, Forrest D et al. American Thyroid Association Guide to investigating thyroid hormone economy and action in rodent and cell models. Thyroid 2014; 24: 88-168 